\title{
Biochemical Signatures of Doppel Protein in Human Astrocytomas to Support Prediction in Tumor Malignancy
}

\author{
Paola Rognoni, ${ }^{1}$ Laurent R. Chiarelli, ${ }^{1}$ Sergio Comincini, ${ }^{2}$ Alberto Azzalin, ${ }^{3,4}$ \\ Clelia Miracco, ${ }^{5}$ and Giovanna Valentini ${ }^{1}$ \\ ${ }^{1}$ Dipartimento di Biochimica, "A. Castellani”, University of Pavia, Via Taramelli 3/b, 27100 Pavia, Italy \\ ${ }^{2}$ Dipartimento di Genetica e Microbiologia, "A. Buzzati-Traverso", University of Pavia, Via Ferrata 1, 27100 Pavia, Italy \\ ${ }^{3}$ IRCCS Policlinico S. Matteo, Via Ferrata 1, 27100 Pavia, Italy \\ ${ }^{4}$ IGM-CNR, Via Ferrata 1, 27100 Pavia, Italy \\ ${ }^{5}$ Dipartimento di Patologia Umana e Oncologia, University of Siena, via Banchi di Sotto 55, 53100 Siena, Italy
}

Correspondence should be addressed to Sergio Comincini, sergio.comincini@unipv.it

and Giovanna Valentini, giovanna.valentini@unipv.it

Received 10 June 2010; Revised 9 September 2010; Accepted 17 September 2010

Academic Editor: Paul Harrison

Copyright (c) 2010 Paola Rognoni et al. This is an open access article distributed under the Creative Commons Attribution License, which permits unrestricted use, distribution, and reproduction in any medium, provided the original work is properly cited.

\begin{abstract}
Doppel (Dpl) is a membrane-bound glycoprotein mainly expressed in the testis of adult healthy people. It is generally absent in the central nervous system, but its coding gene sequence is ectopically expressed in astrocytoma specimens and in derived cell lines. In this paper, we investigated the expression and the biochemical features of Dpl in a panel of 49 astrocytoma specimens of different WHO malignancy grades. As a result, Dpl was expressed in the majority of the investigated specimens (86\%), also including low grade samples. Importantly, Dpl exhibited different cellular localizations and altered glycan moieties composition, depending on the tumor grade. Most low-grade astrocytomas (83\%) showed a membrane-bound Dpl, like human healthy testis tissue, whereas the majority of high-grade astrocytomas (75\%) displayed a cytosolic Dpl. Deglycosylation studies with N-glycosidase F and/or neuraminidase highlighted defective glycan moieties and an unexpected loss of sialic acid. To find associations between glial tumor progression and Dpl biochemical features, predictive bioinformatics approaches were produced. In particular, Decision tree and Nomogram analysis showed well-defined Dpl-based criteria that separately clustered low-and high-grade astrocytomas. Taken together, these findings show that in astrocytomas, Dpl undergoes different molecular processes that might constitute additional helpful tools to characterize the glial tumor progression.
\end{abstract}

\section{Introduction}

Astrocytomas are human brain tumors that arise from astrocytes, the most abundant type of glial cells of the central nervous system, or, more likely, from resident cancer stem cells. These tumors are divided into grades according to their histological characteristics and following the classification guidelines given by the World Health Organization (WHO) [1]. Unfortunately, the pathological classification of astrocytomas is often problematic due to their extensive heterogeneity, thus suffering from the subjectivity of the anatomopathologists. Presently, low-grade astrocytomas include pilocytic (WHO grade I) and diffuse (grade II) astrocytomas, whereas high-grade astrocytomas comprise anaplastic astrocytomas (grade III) and glioblastoma multiforme (GBM, grade IV). GBMs are the most common and aggressive forms of these tumors in adults [2], and despite decades of research on tumor biology, they continue to have a poor prognosis. Median survival time of patients with GBM without treatments is approximately 3 months, whereas it approaches to one year following aggressive surgical and adjuvant therapy, such as radio- and chemotherapy [3]. Therefore, there is a pressing need for the elucidation of molecular mechanisms involved in malignant cell transformation as well as for the development of sensitive and specific biomarkers that enable early and accurate diagnosis and prognostic prediction. In this context, it is worth mentioning that genetic alterations that drive glial cell transformation and malignant progression result in tumor-specific changes in protein expression and/or posttranslational modifications [4-6]. 
Recently, it has been reported that doppel (Dpl), a prion protein family member mainly expressed in testis but generally absent in central nervous system [7], is aberrantly expressed in astrocytic tumors where it displays cellular localization and molecular properties different from those of the corresponding testis protein [8-11]; Dpl in healthy human testis is a glycoprotein of 126 amino acids, bound to the cell surface through a glycosylphosphatidylinositol (GPI) anchor $[12,13]$. Although the cellular function of Dpl has not still unravelled, its involvement in spermiogenesis and in male fertility regulation has been suggested $[14,15]$. The aberrant expression of doppel protein was mainly investigated in established astrocytoma cell lines, highlighting an abnormal retention of the gene transcript within the nucleus and an unusual cytoplasmic localization of the protein [10]. In particular, the protein displayed an increased glycosylation profile and a predominant accumulation within lysosomal vesicles $[10,11]$. Unfortunately, knowledge concerning the molecular features of $\mathrm{Dpl}$ in tumor mostly originates from studies performed on astrocytoma-derived cell lines that, although recognized as a good model for pathogenesis, they could display protein patterns that often do not match the bioptic counterparts. Therefore, a biochemical characterization of $\mathrm{Dpl}$ isolated from a collection of different grades of astrocytoma specimens has been undertaken. This analysis was then coupled with a bioinformatics approach to underline associations between Dpl biochemical and expression features and glial tumor malignancy progression.

\section{Materials and Methods}

2.1. Human Biopsy Samples. Astrocytoma specimens were provided by Dr. Benericetti (Azienda Ospedaliera di Parma, Parma, Italy) and by Prof. Clelia Miracco (University of Siena). These specimens were obtained by surgical resection after informed consent of the patients and immediately stored at $-80^{\circ} \mathrm{C}$. Sections of specimens were histologically and morphologically evaluated by independent neuropathologists and classified according to the $2000 \mathrm{WHO}$ guidelines. Clinical data of the investigated astrocytoma patients $(n=$ 49) are reported in (see Table 1 in Supplementay Material available online at doi:10.1155/2010/301067.) Human nonneoplastic autoptic testis and brain tissues, respectively, as Dpl-positive and Dpl-negative controls, were provided by the "Dipartimento di Medicina Legale e Sanità Pubblica, Università di Pavia, Pavia, Italy”.

2.2. Dpl Extraction and Partial Purification. Microsomal and cytosolic fractions were prepared as previously described [10]. The relative purity of the fractions was checked assaying the activity of both L-lactic dehydrogenase and glucose 6-phosphatase used as marker enzymes [16, 17]. Partial purification of Dpl was performed using a cation exchanger precolumn (HiTrap SP, $1 \mathrm{~mL}$, GE-Healthcare, Piscataway, NJ, USA) arranged on the top of an anion exchanger column (HiTrap Q, $5 \mathrm{~mL}$, GE-Healthcare), equilibrated in $50 \mathrm{mM}$ sodium phosphate $\mathrm{pH} 6.5,1 \mathrm{mM}$ ethylenediamminetetraacetic acid, and $1 \mathrm{mM}$ phenylmethylsulfonyl fluoride.
Elution of bound proteins was performed separately, with a 0 to $1 \mathrm{M} \mathrm{NaCl}$ linear gradient. Purified proteins were then accurately quantified [18], and Dpl was detected by means of $12 \%$ SDS-PAGE and by immunoblotting analysis using a Dpl-specific antiserum (Dpl-Val), previously described in $[10,11]$, and commercial IgG anti-Dpl goat (N-20) and rabbit (FL-176) antisera (Santa-Cruz Biotechnologies).

2.3. Immunohistochemistry Dpl Detection. Immunohistochemistry was performed on $3 \mu \mathrm{m}$-thick sections, from formalin-fixed and paraffin-embedded glioblastoma fragments, using the streptavidin-biotin peroxidase complex method. Sections were dewaxed in Bio-Clear (Bio-Optica, Milano, Italy) and rehydrated with graded ethanol until distilled water. Endogenous peroxidase activity was blocked by treating the slides with $3.0 \%$ hydrogen peroxidase in distilled water for $10 \mathrm{~min}$, followed by three washes in Tris/HCl buffer, $\mathrm{pH}$ 7.5. The antihuman Dpl-Val antibody $(1: 200)$ was applied overnight at $4^{\circ} \mathrm{C}$. Dpl was detected with the UltraVision LP Large Volume Detection System HRP or AP Polymer (Ready-To-Use) (Bio-Optica) and using diaminobenzidine (Dako, Milano, Italy) for $8 \mathrm{~min}$ as chromogen. Sections were then counterstained with Mayer's hematoxylin. Negative controls were performed by replacing the primary antibody with a nonimmune rabbit serum.

2.4. Deglycosylation Assays. Chemical deglycosylation was performed by trifluoromethanesulfonic acid (TFMS) as described in [19]. Briefly, $2 \mathrm{mg}$ (accurately determined as before) of lyophilized protein was dissolved in $300 \mu \mathrm{L}$ of anisole/TFMS $1: 2$ and stirred at $25^{\circ} \mathrm{C}$ for $3 \mathrm{~h}$. The reaction was neutralized by the gradual addition of $100 \mu \mathrm{L}$ pyridine, and the sample was stored at $-20^{\circ} \mathrm{C}$ for $1 \mathrm{~h}$. After addition of trichloroacetic acid at the final concentration of $25 \%$, the samples were centrifuged, twice washed with acetone and subjected to $15 \%$ SDS-PAGE and immunoblotting analysis.

Enzymatic deglycosylation was carried out using peptide: N-glycosidase F (PNGase F) or/and neuraminidase (New England Biolabs, Ipswich, MA, USA) according to the manufacturer. Briefly, $100 \mu \mathrm{g}$ of protein was heat denaturated for $10 \mathrm{~min}$ in the presence of $0.5 \%$ SDS and $1 \% \beta$ mercaptoethanol. After an overnight incubation at $37^{\circ} \mathrm{C}$ with $50 \mathrm{mM}$ sodium phosphate $\mathrm{pH} 7.5$ and 1\% NP-40 in the presence of $1,000 \mathrm{U}$ of PNGase F or/and $100 \mathrm{U}$ of neuraminidase, the reaction was stopped with trichloroacetic acid, and the proteins were analyzed by $12 \%$ SDS-PAGE and immunoblotting.

2.5. Two-Dimensional Polyacrylamide Gel Electrophoresis (2D-PAGE). 2D-PAGE was performed using Immobiline DryStrip pH 3-10 and IPG Buffer 3-10 (GE Healthcare) as described in [20]. Proteins $(60 \mu \mathrm{g})$ were water dialyzed, lyophilized, and resuspended in $200 \mu \mathrm{L}$ of $8 \mathrm{M}$ urea, $2 \%$ CHAPS, 2\% IPG Buffer 3-10, and applied to the DryStrips via the rehydration technique. Strips were focused on a Multiphor II system (GE Healthcare) at $20^{\circ} \mathrm{C}$ with the following program: $100 \mathrm{~V}$ for $6 \mathrm{~h}, 300 \mathrm{~V}$ for $5 \mathrm{~h}, 600 \mathrm{~V}$ for $2 \mathrm{~h}, 1000 \mathrm{~V}$ for $1 \mathrm{~h}$, and $3000 \mathrm{~V}$ for $3 \mathrm{~h}$. After isoelectric 
focusing, strips were incubated $30 \mathrm{~min}$ in $50 \mathrm{mM}$ Tris $/ \mathrm{HCl}$, $\mathrm{pH} 8.8,6 \mathrm{M}$ Urea, $30 \%(\mathrm{v} / \mathrm{v})$ glycerol, $2 \%(\mathrm{w} / \mathrm{v})$ SDS, $0.1 \%$ $(\mathrm{w} / \mathrm{v})$ bromophenol blue, layered onto a $12 \%$ SDS-PAGE, and immunoblotted as before.

2.6. Statistical and Bioinformatics Analysis. To calculate the significance of microsomal-cytosolic distribution of $\mathrm{Dpl}$ among WHO grades, an Anova one-ways test was adopted, considering as significant a $P$ value less than .05. The Decision tree and the Nomogram analysis were performed using the Orange data mining software (http://www.ailab.si/orange/). The molecular weight of Dpl isoforms was calculated from molecular weights standards. For densitometric evaluations, a relative scale from 1 to 10 values of Dpl quantity (in arbitrarily units) was assessed. For Nomograms, interval confidence of Probabilities $(P)$ was assessed at $P=.95$.

\section{Results}

3.1. Dpl Expression in Astrocytoma Specimens. To characterize $\mathrm{Dpl}$ expression in astrocytomas, 49 bioptic specimens, graded according to the World Health Organization (WHO) criteria [1], were mechanically lysed and fractionated to obtain membrane-bound (microsomal) and/or soluble (cytoplasmic) proteins. The relative purity of microsomal and cytoplasmic fractions of all specimens was checked assaying the activity of both L-lactic dehydrogenase and glucose 6-phosphatase marker enzymes. L-lactic dehydrogenase activity was found only in the cytosolic fractions, whereas glucose 6-phosphatase activity was mostly present in the microsomal ones (more than 95\%), thus exhibiting only a minimal percent in the cytosolic fraction. Taken together, these data supported the good quality of both preparations for each sample analyzed.

Along with their clinical features, clinical cases included WHO grades I (2 samples), II (5), III (7), and IV (35) (Supplementary Table 1). Human nonneoplastic controls from autoptic testis and brain tissues ( $n=2$ each) were also fractionated, and Dpl expression was evaluated. As expected, Dpl was undetectable in both cytosolic and microsomal fractions of brain (data not shown) as well as in testis cytoplasmic fractions (Figure 1(a)). Conversely, Dpl was visualized as a single band of approximately $31 \mathrm{kDa}$ in the microsomal fraction of testis tissue (Figure 1(b)). In astrocytomas, Dpl was detected in the majority of the analyzed specimens $(42 / 49,86 \%)$. Differently, Dpl was undetectable in one WHO grade I and in three WHO grade III and IV samples (data not shown).

As reported in Figure 1, Dpl was mostly expressed in the soluble fraction of the specimens $(38 / 42,90 \%)$. Moreover, the presence of the soluble $\mathrm{Dpl}$ alone was significantly associated with high- compared to low-grade samples (Figure 1(c)). Ten specimens endowed with cytoplasmic Dpl exhibited the protein also in the microsomal fraction (Figure 1(b), samples 1, 2, 3, 17, 23, 24, 27, 29, 32, 33), whereas four specimens showed the protein exclusively in the microsomal fraction (Figure 1(b), samples 39-42). Interestingly, $83 \%$ of grade I and II tumors were characterized by the presence of the membrane-bound Dpl, whereas only $25 \%$ of high-grade samples exhibited this molecular form (Figure 1(d)). Moreover, whereas cytoplasmic fractions, after immunoblotting, showed two or more Dpl isoforms (Figure 1(a)), Dpl isolated from microsomal fractions generally showed a single isoform of approximately $31 \mathrm{kDa}$ (Figure 1(b)). The authenticity as well as the reproducibility of positive bands was confirmed by subjecting to immunoblotting experiments, both microsomal and soluble fractions of three astrocytoma samples (one low-grade and two high-grade), and using three different primary antibodies (i.e., Dpl-Val, N-20, and FL-176). A Dpl-positive (testis) and a Dpl-negative (healthy brain) control were also assayed (Supplementary Figure 1).

Differences in Dpl subcellular localization were also highlighted by immunohistochemistry in different glioblastoma sections, showing a predominantly extensive cytoplasmic localization, whereas a membrane immunostaining was less frequently exhibited (Figures 2(a) and 2(b)). As expected $[9,10,12]$, in the cortex section of peritumoral area, Dpl expression was faint compared to the neoplastic tissue (Figure 4(c)).

\subsection{Biochemical Characterization of $D p l$ in Astrocytomas.} Previously, it has been reported that Dpl protein in healthy human testis is a glycoprotein, with two N-linked and one O-linked oligosaccharide [14]. To compare glycan moieties composition of Dpl in testis (microsomal fraction) and in astrocytoma specimens (soluble fraction), chemical and enzymatic deglycosylation studies were performed, after a partial purification by ion-exchange chromatography. Because of the limited available amounts in low-grade samples, four high-grade samples (i.e., 9, 10, 15, 23) were subjected to this biochemical characterization. After chemical deglycosylation with TFMS treatment that removes total sugars from glycoproteins and immunoblotting, in glial tumor and in testis, Dpl migrated as a $15 \mathrm{kDa}$ protein (Figure 3(a)), in accordance with the expected molecular mass of the polypeptide chain $[14,21]$, this also proved the integrity of Dpl in the specimens, following the above mentioned treatments.

After PNGase F treatment that specifically digested the $\mathrm{N}$-linked glycan moieties, testis Dpl exhibited a $22 \mathrm{kDa}$ protein (Figure 3(b)), indicating the removal of approximately $9 \mathrm{kDa}$ of $\mathrm{N}$-linked sugar chains. As a result, testis $\mathrm{Dpl}$ turned out to have a nearly $7 \mathrm{kDa}$ O-bound oligosaccharide. Moreover, after a digestion with neuraminidase that removes the terminal sialic acid residues, testis Dpl migrated as a $28 \mathrm{kDa}$ protein, indicating that the total sialic acid mass removed consisted of approximately $3 \mathrm{kDa}$ in mass. Lastly, a digestion with neuraminidase in association with PNGase lowered the band to about $20 \mathrm{kDa}$, suggesting that the amount of sialic acid lost exclusively by the O-ligated moiety of testis $\mathrm{Dpl}$ amounted to nearly $2 \mathrm{kDa}$ (Figure $3(\mathrm{~b})$ ). Unfortunately, digestion with O-glycosidase that removes O-linked glycan moiety was unsuccessful (data not shown), preventing us from corroborating data on oligosaccharide distribution.

Dpl cytoplasmic fractions in astrocytoma specimens generally exhibited two or more bands with a molecular mass ranging from 20 to $90 \mathrm{kDa}$ (Figure 1(a)). When 


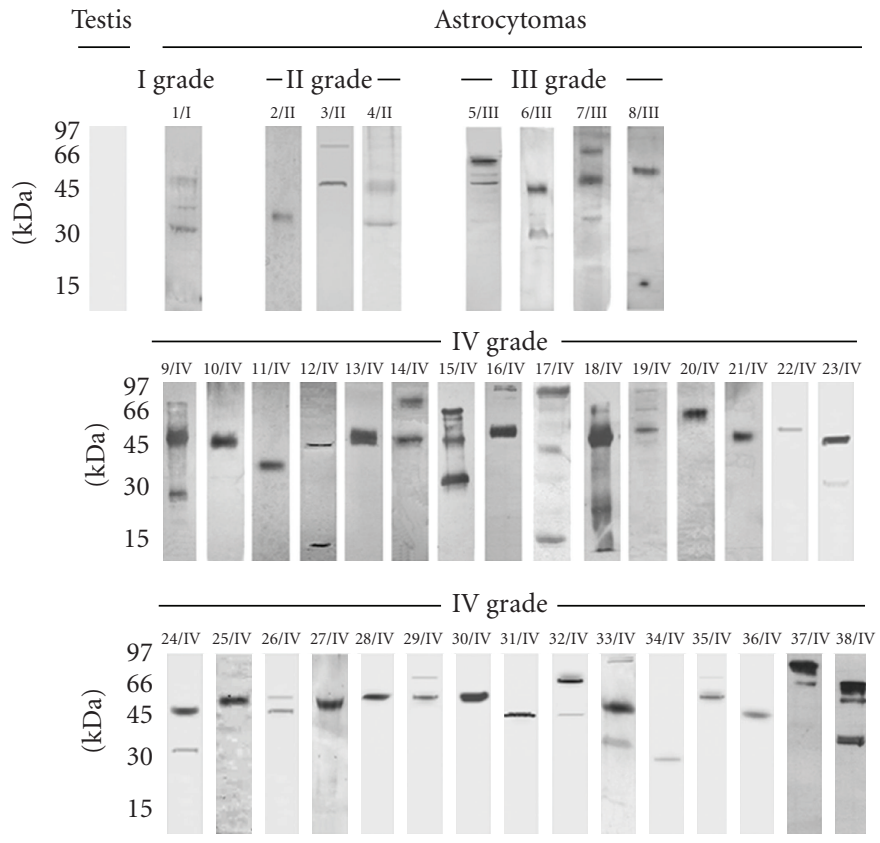

(a)

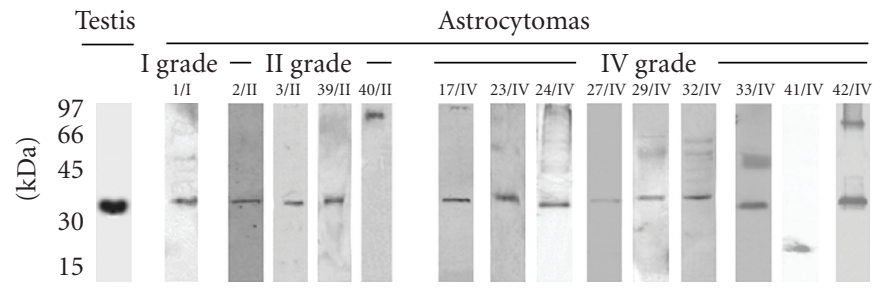

(b)

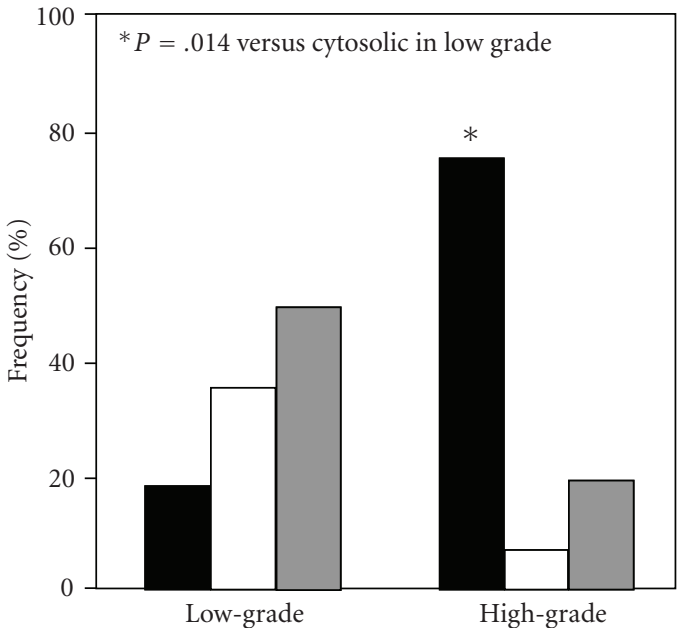

(c)

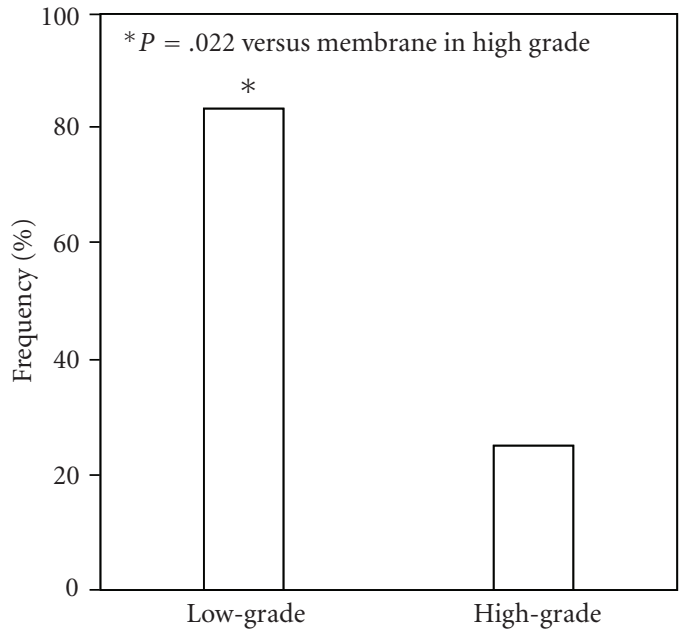

(d)

FIGURE 1: Identification and cellular localization of Dpl in testis and astrocytoma samples. Immunoblotting analysis of cytoplasmic (a) and microsomal (b) fractions. To limit the figure size, only positive samples have been reported. Dpl was detected with the anti-Dpl rabbit antiserum (Dpl-Val) previously described in $[10,11]$, loading $20 \mu \mathrm{g}$ of purified proteins. Each astrocytoma sample is identified by a progressive number followed by their WHO grade of malignancy (1/I, 2/II, etc.). (c) Distribution of Dpl in low- and high-grade astrocytoma samples as a cytoplasmic fraction (black bars), microsomal fraction (white bars) and in both fractions (grey bars). (d) Distribution of microsomal Dpl in low- and high-grade astrocytoma samples, regardless of the presence/absence of the cytosolic protein. Asterisks indicated statistically significant Anova One-way probabilities $(P)$. 


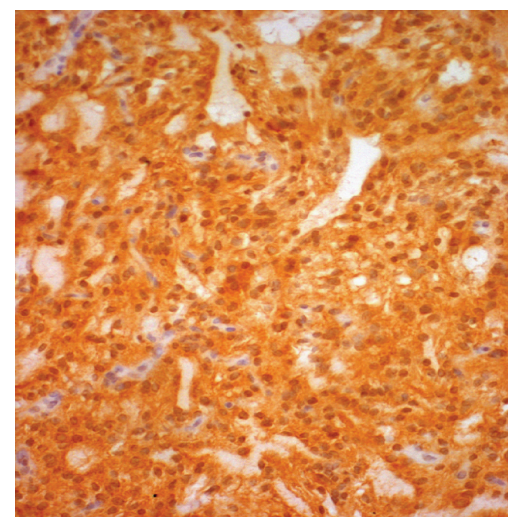

(a)

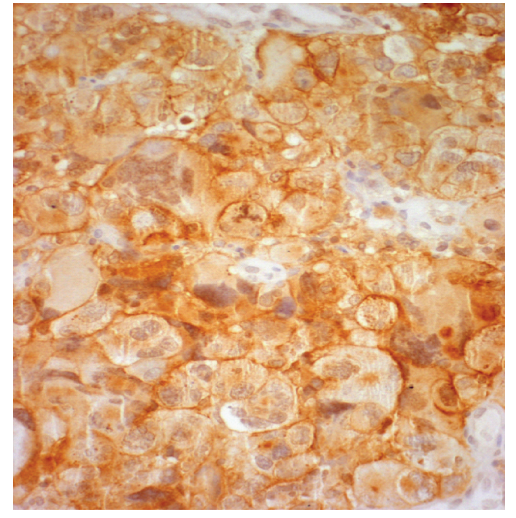

(b)

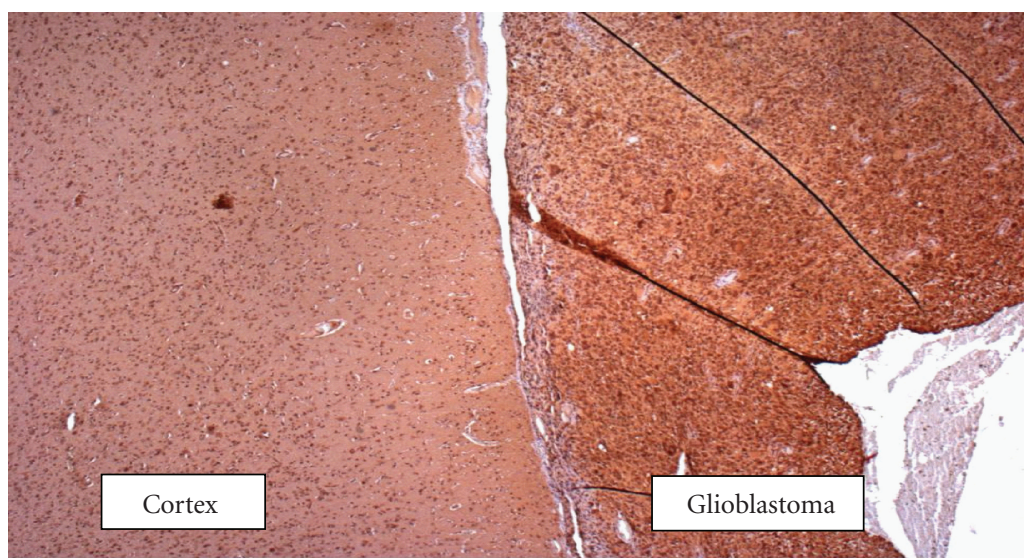

(c)

Figure 2: Dpl immunohistochemistry localization in glioblastoma and peritumoral cortex sections. Dpl was detected using Dpl-Val antibody. Panels (a) and (b) represent sections of two different glioblastomas, one of which showing a marked Dpl immunoreactivity in the cytoplasm (Panel (a)), and the other at the plasma membrane (Panel (b)) (original magnification 200x). A low Dpl immunoreactivity is exhibited by the peritumoral cortex (Panel (c), left), compared to the glioblastoma area (Panel (c), right) (original magnification 25x).

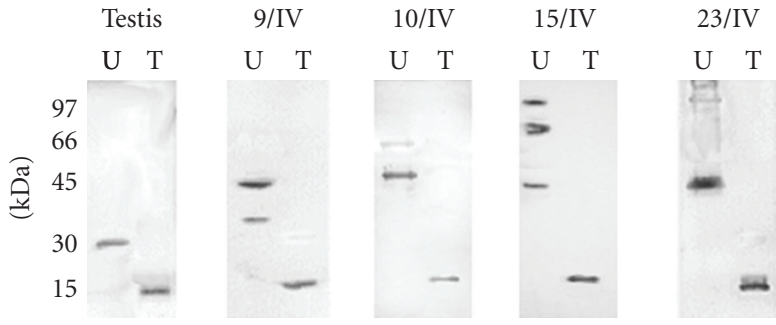

(a)

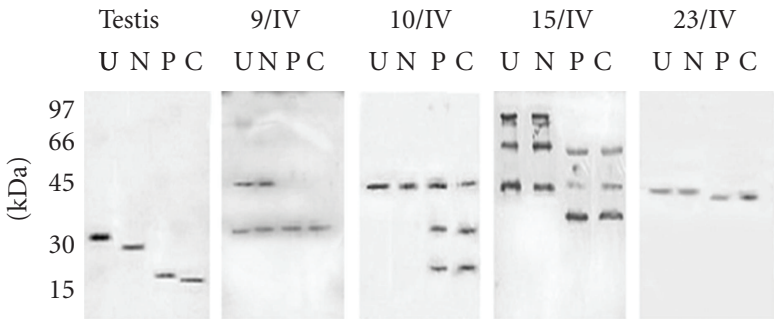

(b)

Figure 3: Deglycosylation patterns of healthy human testis and glial tumor Dpl. Testis (microsomal fraction) and astrocytomas (soluble fraction) glycoforms of Dpl were analyzed by chemical deglycosylation with TFMS (a) and by enzymatic deglycosylation with neuraminidase and/or PNGase F (b), followed by immunoblotting using a Dpl-specific polyclonal antibody [10, 11]. U: untreated; T: TFMS treatment; N: neuraminidase digestion; P: PNGase F digestion; C: neuraminidase plus PNGase F digestion.

cytoplasmic protein was digested with PNGase F, complex electrophoretic patterns were obtained, suggesting a high heterogeneity of the glycan moieties, even within the same specimen (Figure 3(b)). On the other hand, the digestion with neuraminidase left the molecular mass of all isoforms unchanged, indicating that soluble Dpl was likely devoid of sialic acid. The lack of an acidic component in the soluble Dpl molecule was also evidenced through 2D-PAGE Dpl protein analysis: all Dpl soluble isoforms exhibited isoelectric point values $(\mathrm{pI}=5.9-6.5)$ higher than that of 


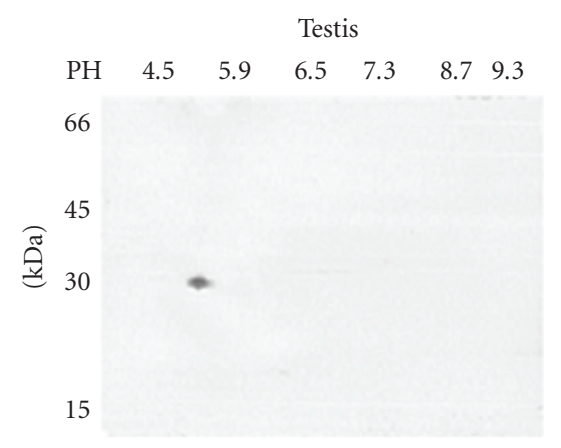

(a)

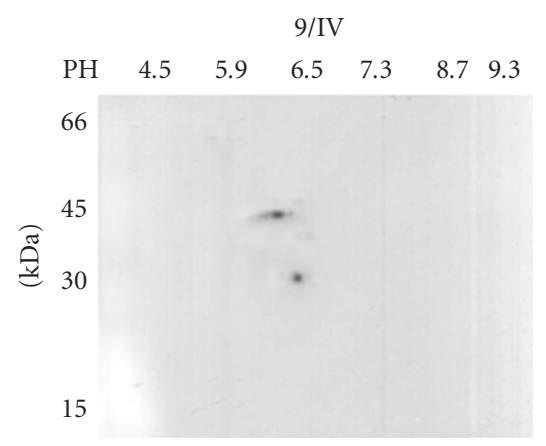

(b)

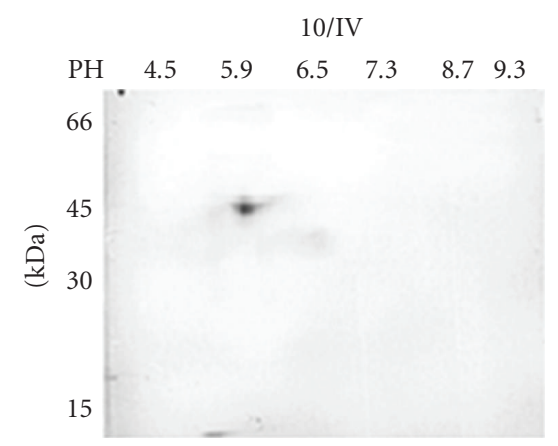

(c)

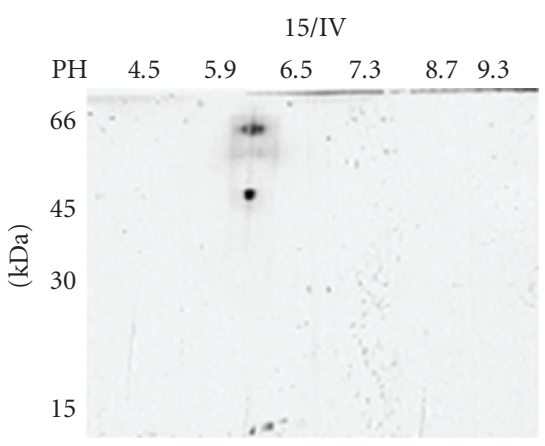

(d)

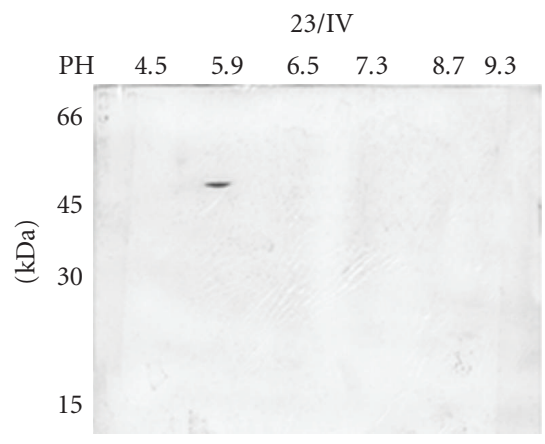

(e)

FIGURE 4: Isoelectric point determination of Dpl protein in nonneoplastic human testis and in glioblastoma multiforme (GBM) samples. 2D-PAGE was performed using $60 \mu \mathrm{g}$ of purified protein samples on a linear immobilized $\mathrm{pH}$ gradient (i.e., $\mathrm{pH} 3-10$ ), followed by a $12 \%$ SDS-PAGE. Dpl was then detected by immunoblotting as reported above.

the Dpl isolated testis protein $(\mathrm{pI}=5.3)$, as illustrated in Figure 3.

3.3. Bioinformatics Analysis. Then, Dpl expression and biochemical features were tentatively correlated with glial tumor progression using a bioinformatics-derived approach; Dpl expression patterns were catalogued according to Dpl relative densitometric expression (RQ, adopting a scale from 1 to 10 arbitrary units), to the cellular topology (i.e., Dpl expressed in microsomal fractions, cytosolic fractions, or both), to the apparent molecular mass of Dpl isoforms, to the $2000 \mathrm{WHO}$ tumor malignancy grading criteria, and finally, according to the anatomical localization of the tumor specimens (Supplementary Table 1). After a Decision tree analysis, Dplpositive tumor samples were roughly subdivided into two main branches, according to the molecular mass of Dpl isoforms (Figure 5): for Dpl masses lower than $47.5 \mathrm{kDa}$, low-grade astrocytoma samples (i.e., WHO grades I and II) were clustered; in this group, a minority of WHO grade IV samples $(9 / 32,28 \%)$ were also identified. Differently, the majority of WHO IV specimens $(23 / 32,72 \%)$ clustered at $\mathrm{Dpl}$ mass higher than $47.5 \mathrm{kDa}$. Importantly, all anaplastic astrocytoma classified samples (WHO III) were distributed in this branch, according to: (i) a Dpl molecular weight higher than $74 \mathrm{kDa}$, (ii) a Dpl RQ minor or equal to 6.5 , and (iii) to a Dpl cytosolic localization of the protein. To further confirm this partition, tumor specimens were further grouped in two main classes, low-grade (WHO I and II) and high-grade astrocytoma (WHO grade III and IV) and subjected to a Nomogram analysis to evaluate the performance of the above-mentioned Dpl-related molecular and cellular criteria in distinguishing tumor-grading progression. This analysis, reported in Figure 6, highlighted that the highest probabilities $(P=.95)$ to sort the samples according to the two main target classes (i.e., low-and high-grade astrocytomas) were obtained in correspondence to lower Dpl mass and RQ and to a microsomal localization for low grade samples: On the contrary, the highest probability to correctly identify high-grade samples was associated with high Dpl molecular weights and RQ and to a cytoplasmic protein localization, as evidenced in the previous Decision tree analysis. As expected, the anatomical localization of the tumor specimens, as well as their age and gender, did not influence the estimation of the probabilities for the two classes.

\section{Discussion}

Previous studies performed on the aberrant expression of doppel (PRND) gene in astrocytoma bioptic specimens and in derived-astrocytoma cell lines showed that transcripts levels were associated with glial tumor progression $[8,9]$. To ascertain whether the expression of doppel (Dpl) protein 


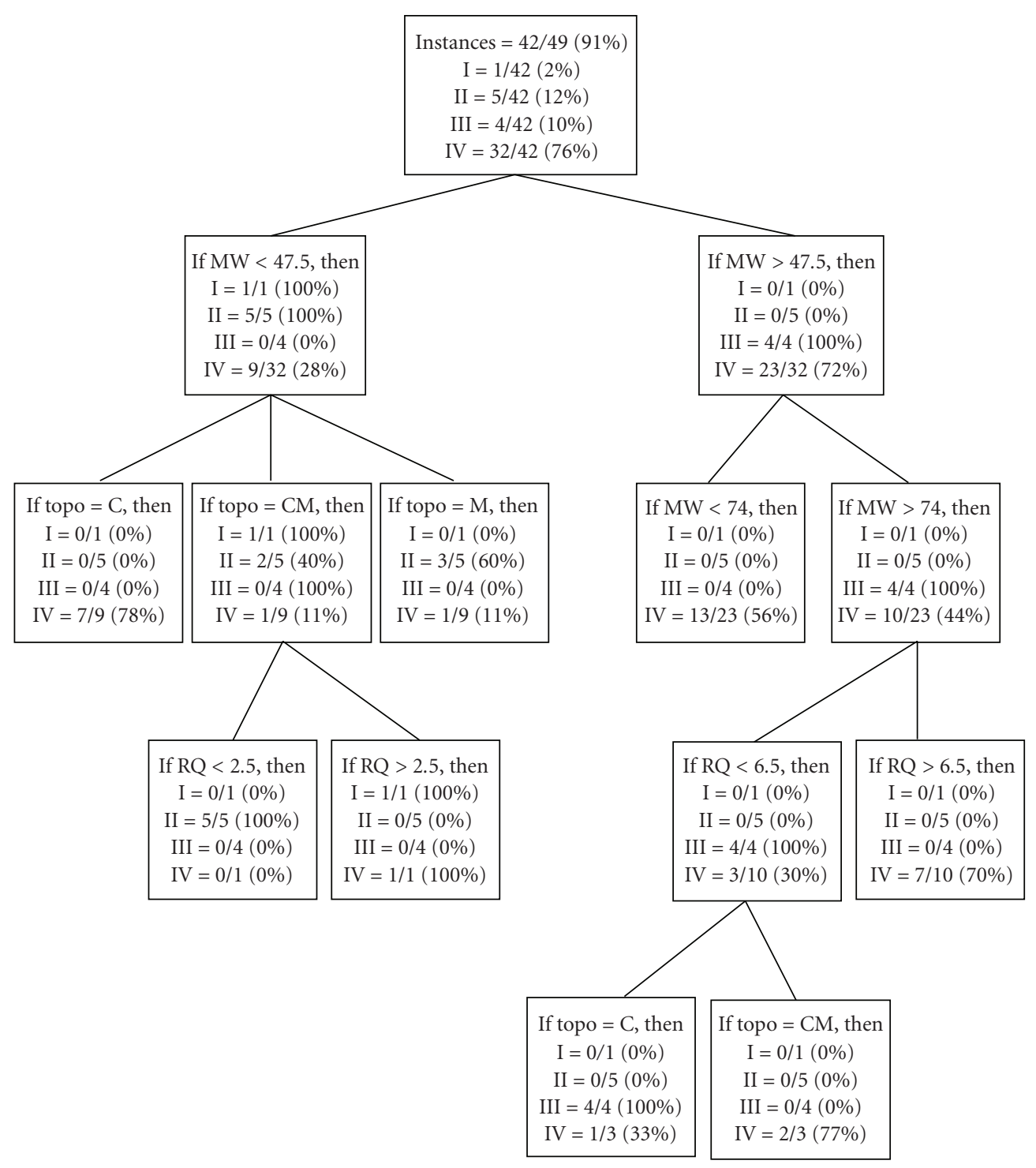

FIgure 5: Decision tree analysis according to Dpl characterization in astrocytoma specimens. The adopted Dpl-based criteria at the decision nodes are reported (i.e. molecular mass of Dpl isoforms, MW; relative quantity of Dpl expression, RQ; Dpl topology, C=cytosol, $\mathrm{M}=$ microsomal or $\mathrm{CM}=$ both $)$. WHO grading and sample percentages are indicated.

might also be associated with the glial tumor grading progression, we performed for the first time a biochemical characterization of $\mathrm{Dpl}$ in a collection of astrocytoma specimens, deriving a predictive bioinformatics model to ascertain the malignancy grade of these tumors.

At a molecular level, Dpl turned out to be expressed in nearly all tumor specimens investigated (86\%) also including low-grade astrocytomas. This suggested that Dpl expression might represent one of the primitive alterations that accompanies the malignant transformation in astrocytomas. According to our results, this tumor grading transition in astrocytomas was much more depicted considering Dpl topologies within the specimens: Dpl shifted from a microsomal-membrane-associated localization in low-grade samples to a predominant cytoplasmic distribution in anaplastic astrocytoma and in glioblastoma multiforme samples. Similarly, this cytosolic localization of Dpl was evidenced also in astrocytoma-derived cell lines [10].

From a biochemical point of view, microsomal Dpl in astrocytomas was similar to the corresponding testis protein. Thus, Dpl, at the onset of the disease, is likely to be anchored to the outer leaflet of the plasma membrane, through its GPI moiety. Afterwards, as the tumor progresses, $\mathrm{Dpl}$ could fail to anchor to the membrane and localize within the cytoplasm. The reason for this behaviour is presently puzzling, also in the light of our previous results that excluded mutations in the GPI attachment site of the protein [10]. On the other hand, chemical deglycosylation performed on cytoplasmic Dpl led to a protein of $15 \mathrm{kDa}$, the same value displayed by the microsomal Dpl similarly treated. Thus, the aberrant localization of $\mathrm{Dpl}$ should not be 


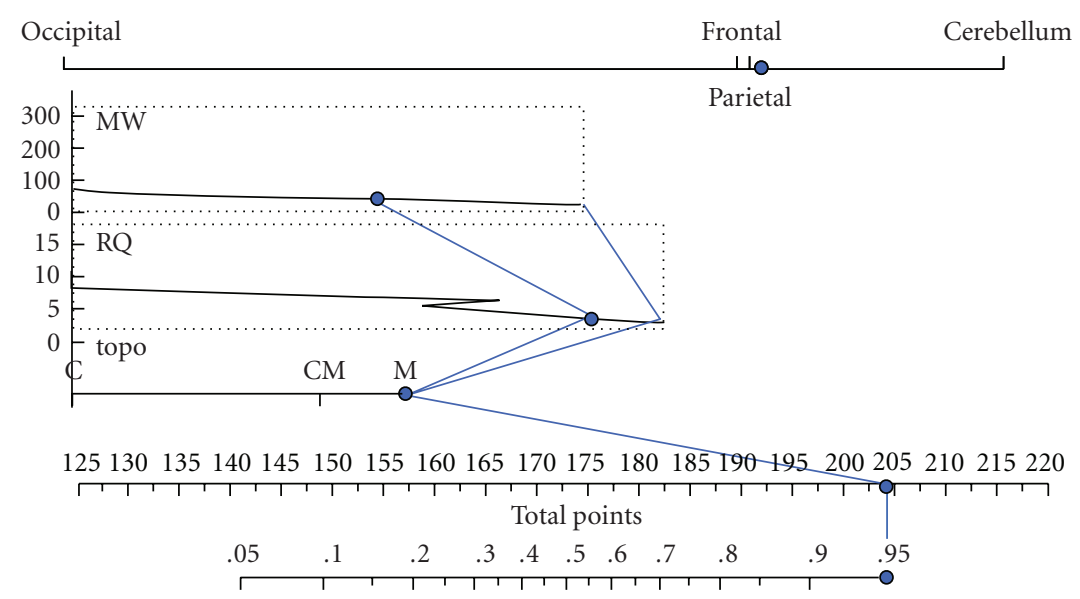

$P$

(a) WHO low-grade

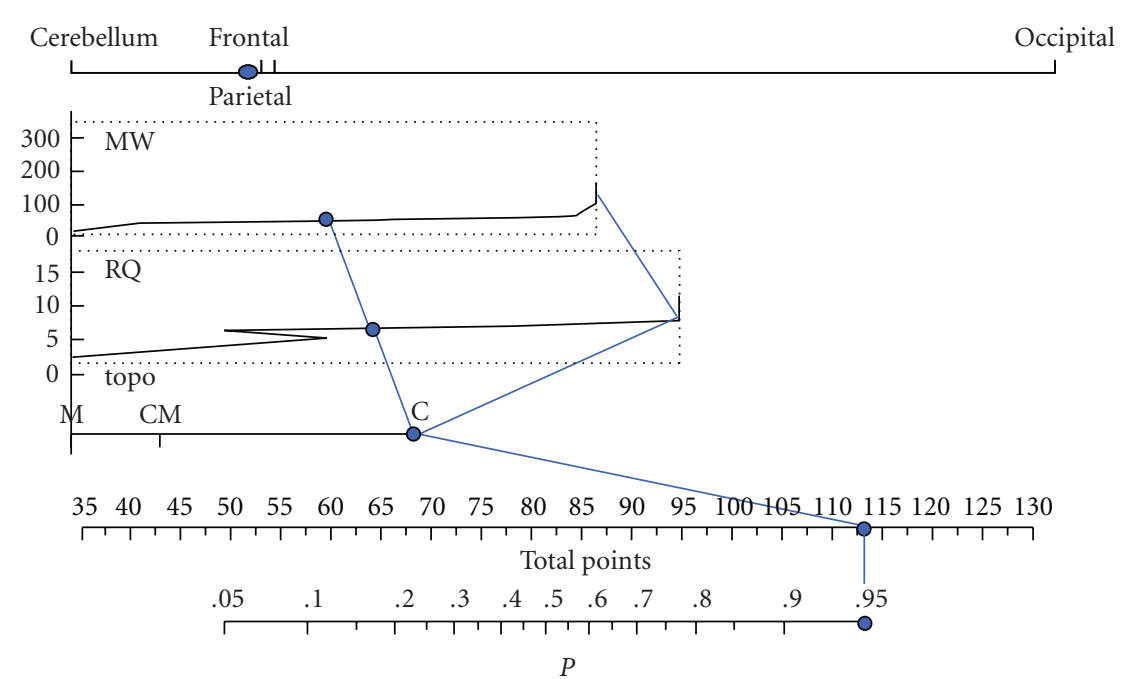

(b) WHO high-grade

FIGURE 6: Nomogram association analysis between Dpl features and astrocytomas. Astrocytoma specimens, grouped in low-(WHO I and II) and high-grade (WHO III and IV), were then associated with Dpl molecular mass (MW), relative quantity (RQ), and topology (topo, $\mathrm{C}$ : cytosol, $\mathrm{M}$ : microsomal, and $\mathrm{CM}$ : both) data. Continuous line-delineated sectors corresponded to target probabilities for low-or high grading sorting of $P=.95$. Total points indicated the sum of the points for each variable.

ascribed to alterations of the polypeptide chain, but rather to some unusual posttranslational modifications. Actually, the presence of $\mathrm{Dpl}$ in the cytosol was mostly associated to an increase of the protein molecular mass and to the number of its isoforms. Therefore, we hypothesized that the soluble Dpl was endowed with heterogeneous, more complex, and larger glycan moieties, even within the same sample. As a matter of fact, enzyme deglycosylation assays agreed with this hypothesis. In some cases, PNGase showed to be able to digest the astrocytoma soluble $\mathrm{Dpl}$, although to a different extent (Figure 2(b), samples 9 and 10), whereas in others, it left the protein unchanged (sample 23). Notably, even the isoforms of the same specimen were found to be differently digested (sample 9), suggesting that the tumor Dpl might contain a defective glycan moiety, possibly due to an impairment of the cell glycosylation machinery. In addition, it is difficult to ascertain whether the failure of PNGase to digest Dpl was related to an actual lack of N-glycosylated component or to a steric hindrance created by these larger glycan moieties. Moreover, the presence of an uncommon fucose in the proximity of the $\mathrm{N}$-glycosylation sites cannot be excluded [4]. Certainly, the cytosolic Dpl was characterized by unusual glycosylation processes. Unluckily, the present data do not allow to unravel the actual distribution of $\mathrm{N}$ - and O-glycan components in Dpl isoforms and to decipher the molecular moieties accountable for the high Dpl molecular mass. By all means, all Dpl astrocytoma isoforms turned out to be devoid of sialic acid, as previously documented in astrocytoma-derived cell lines [10]. The alteration of sialylation pattern is not a novelty in malignancy, although generally the extent of sialylation results was increased rather than lowered in tumors [22]. Thus, the loss of sialic acid in 
astrocytoma Dpl seems a peculiar biochemical feature that might be further investigated.

Different bioinformatics analyses were performed to better elucidate if the above-mentioned Dpl protein signatures might be associated with the glial tumor grading progression. Initially, a supervised approach, based on the assumption that the grouping (i.e., the histological tumor diagnoses made by three independent neuropathologists) is correct, was applied, excluding specimens with ambiguous grading classifications. Finally, adopting the Orange Bioinformatics platform, led to the definition of a Decision tree that straightened into different branches low-versus-high-grade astrocytomas, based mostly on the molecular masses of $\mathrm{Dpl}$ isoforms. Of note, three decision nodes (i.e., molecular weight $>74 \mathrm{kDa}, \mathrm{RQ}<6.5$ and a cytoplasmic localization of $\mathrm{Dpl}$ isoforms) subgrouped anaplastic astrocytomas within the group of glioblastoma multiforme specimens. Naïve Bayesan-based Nomograms further highlighted that lowand high-grade astrocytomas had complementary distributions in terms of microsomal and cytoplasmic localization of $\mathrm{Dpl}$ isoforms, the former also characterized by lower expression and low molecular weight of $\mathrm{Dpl}$ isoforms.

In conclusion, a biochemical characterization and a bioinformatics analysis of $\mathrm{Dpl}$ expression in a panel of astrocytoma specimens allowed to parallel molecular signatures to the glial tumor malignancy grading progression. Despite Dpl potential usefulness in diagnosis, the function of this protein in glial tumor progression remains elusive and worthy of further studies.

\section{Acknowledgments}

This paper was supported by grants from the University of Pavia "Fondi per la Ricerca di Ateneo" and from the Italian MIUR "Progetti di Ricerca di Rilevante Interesse Nazionale". The authors are grateful to Dr. Eugenio Benericetti (Azienda Ospedaliera di Parma) and to Dr. Yao Chen (Dipartimento di Medicina Legale e Sanità Pubblica, Università di Pavia) for providing bioptic and autoptic samples. P. Rognoni and L. R. Chiarelli are contributed equally to this work.

\section{References}

[1] P. Kleihues and W. K. Cavenee, Eds., World Health Organization Classification of Tumours of The Nervous System, IARC Press, Lyon, France, 2000.

[2] H. Ohgaki and P. Kleihues, "Genetic pathways to primary and secondary glioblastoma," American Journal of Pathology, vol. 170, no. 5, pp. 1445-1453, 2007.

[3] C. Dai and E. C. Holland, "Glioma models," Biochimica et Biophysica Acta, vol. 1551, no. 1, pp. M19-M27, 2001.

[4] T. F. Ørntoft and E. M. Vestergaard, "Clinical aspects of altered glycosylation of glycoproteins in cancer," Electrophoresis, vol. 20, no. 2, pp. 362-371, 1999.

[5] R. Chen, J. R. Eshleman, R. A. Brodsky, and M. E. Medof, "Glycophosphatidylinositol-anchored protein deficiency as a marker of Mutator phenotypes in cancer," Cancer Research, vol. 61, no. 2, pp. 654-658, 2001.

[6] T. W. Vogel, Z. Zhuang, J. Li et al., "Proteins and protein pattern differences between glioma cell lines and glioblastoma multiforme," Clinical Cancer Research, vol. 11, no. 10, pp. 3624-3632, 2005.

[7] R. C. Moore, I. Y. Lee, G. L. Silverman et al., "Ataxia in prion protein $(\mathrm{PrP})$-deficient mice is associated with upregulation of the novel PrP-like protein doppel," Journal of Molecular Biology, vol. 292, no. 4, pp. 797-817, 1999.

[8] S. Comincini, V. Ferrara, A. Arias et al., "Diagnostic value of PRND gene expression profiles in astrocytomas: relationship to tumor grades of malignancy," Oncology Reports, vol. 17, no. 5, pp. 989-996, 2007.

[9] S. Comincini, A. Facoetti, I. Del Vecchio et al., "Differential expression of the prion-like protein doppel gene (PRND) in astrocytomas: a new molecular marker potentially involved in tumor progression," Anticancer Research, vol. 24, no. 3A, pp. 1507-1517, 2004.

[10] S. Comincini, L. R. Chiarelli, P. Zelini et al., "Nuclear mRNA retention and aberrant doppel protein expression in human astrocytic tumor cells," Oncology Reports, vol. 16, no. 6, pp. 1325-1332, 2006.

[11] E. Sbalchiero, A. Azzalin, S. Palumbo et al., "Altered cellular distribution and sub-cellular sorting of doppel (Dpl) protein in human astrocytoma cell lines," Cellular Oncology, vol. 30, no. 4, pp. 337-347, 2008.

[12] G. L. Silverman, K. Qin, R. C. Moore et al., "Doppel is an Nglycosylated, glycosylphosphatidylinositol-anchored protein: expression in testis and ectopic production in the brains of Prnp (0/0) mice predisposed to purkinje cell loss," Journal of Biological Chemistry, vol. 275, no. 35, pp. 26834-26841, 2000.

[13] A. Behrens, "Physiological and pathological functions of the prion protein homologue Dpl," British Medical Bulletin, vol. 66, pp. 35-42, 2003.

[14] K. Peoc'h, C. Serres, Y. Frobert et al., "The human "prionlike" protein Doppel is expressed in both sertoli cells and spermatozoa," Journal of Biological Chemistry, vol. 277, no. 45, pp. 43071-43078, 2002.

[15] A. Behrens, N. Genoud, H. Naumann et al., "Absence of the prion protein homologue Doppel causes male sterility," EMBO Journal, vol. 21, no. 14, pp. 3652-3658, 2002.

[16] A. Kornberg, "Lactic dehydrogenase of muscle : Pyruvate + DPNH Lactate + DPN," in Methods in Enzymology, vol. 1, pp. 441-443, Academic Press, New York, NY, USA, 1955.

[17] R. C. Nordlie and W. J. Arion, "Glucose-6-phosphatase," in Methods in Enzymology, vol. 9, pp. 619-625, Academic Press, New York, NY, USA, 1966.

[18] O. H. Lowry, N. J. Rosebrough, A. L. Farr, and R. J. Randall, "Protein measurement with the Folin phenol reagent," The Journal of Biological Chemistry, vol. 193, no. 1, pp. 265-275, 1951.

[19] A. S. B. Edge, C. R. Faltynek, L. Hof, L. E. Reichert Jr., and P. Weber, "Deglycosylation of glycoproteins by trifluoromethanesulfonic acid," Analytical Biochemistry, vol. 118, no. 1, pp. 131-137, 1981.

[20] A. Görg, W. Weiss, and M. J. Dunn, "Current two-dimensional electrophoresis technology for proteomics," Proteomics, vol. 4, no. 12, pp. 3665-3685, 2004.

[21] H. Mo, R. C. Moore, F. E. Cohen et al., "Two different neurodegenerative diseases caused by proteins with similar structures," Proceedings of the National Academy of Sciences of the United States of America, vol. 98, no. 5, pp. 2352-2357, 2001.

[22] J. W. Dennis, M. Granovsky, and C. E. Warren, "Glycoprotein glycosylation and cancer progression," Biochimica et Biophysica Acta, vol. 1473, no. 1, pp. 21-34, 1999. 

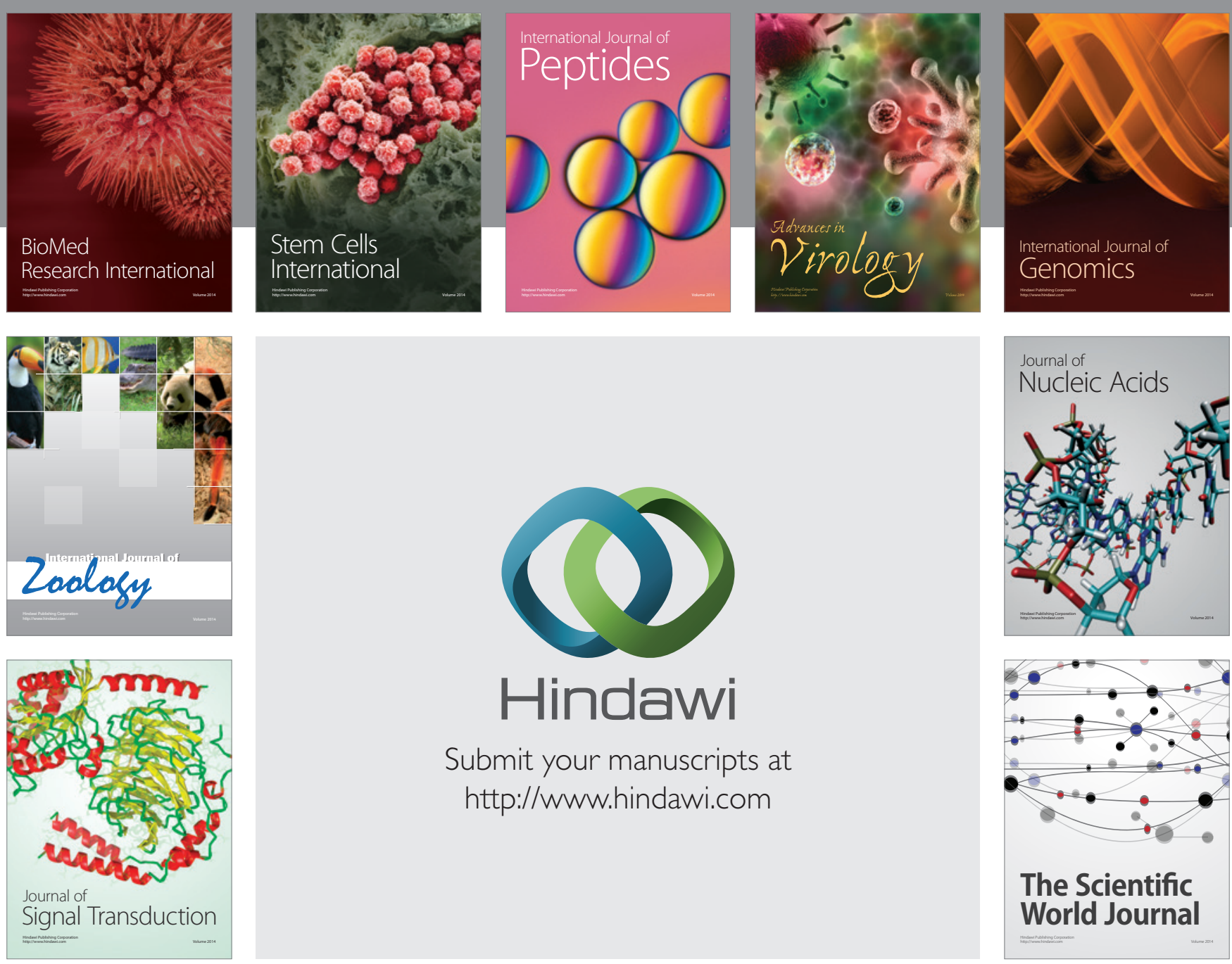

Submit your manuscripts at

http://www.hindawi.com
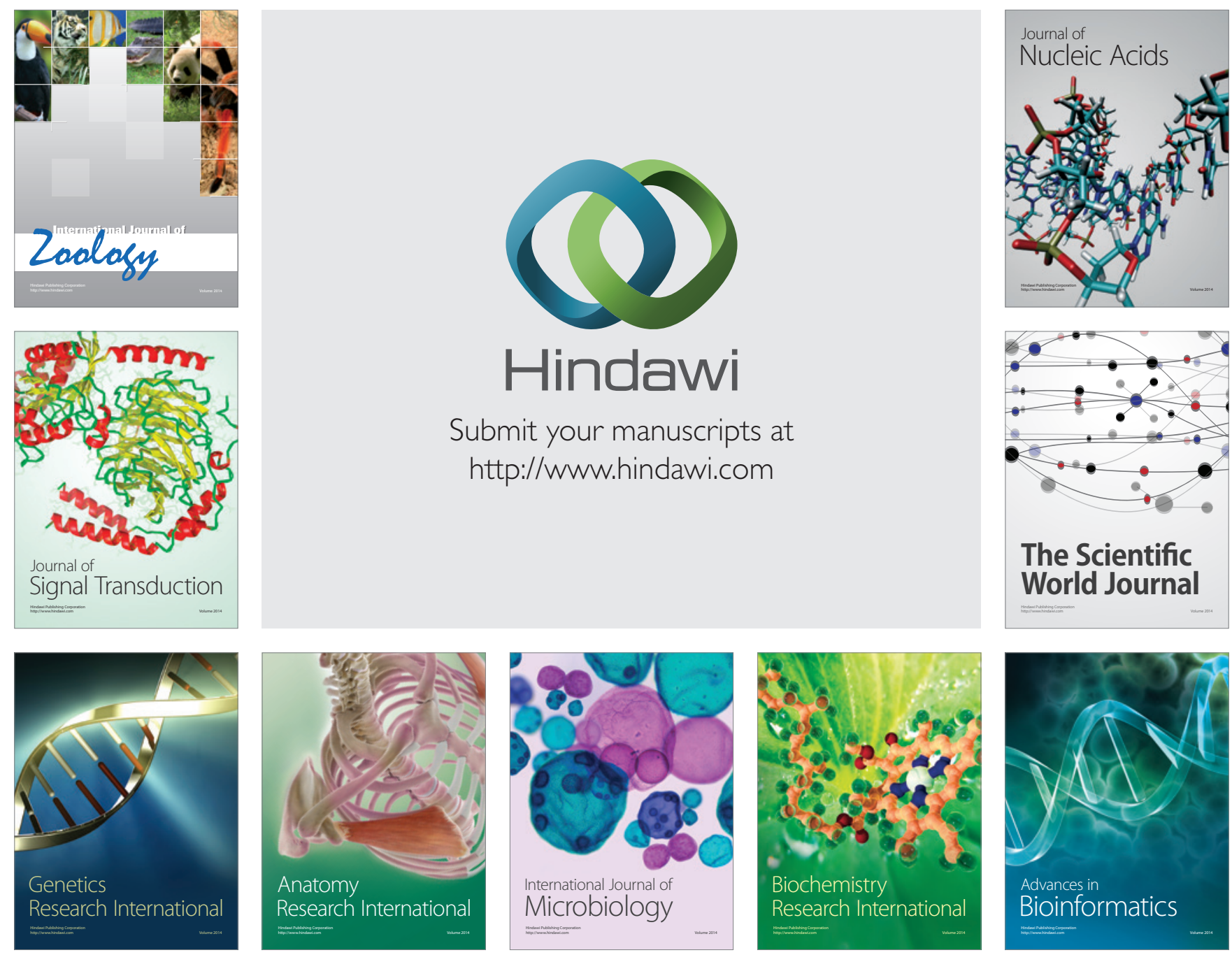

The Scientific World Journal
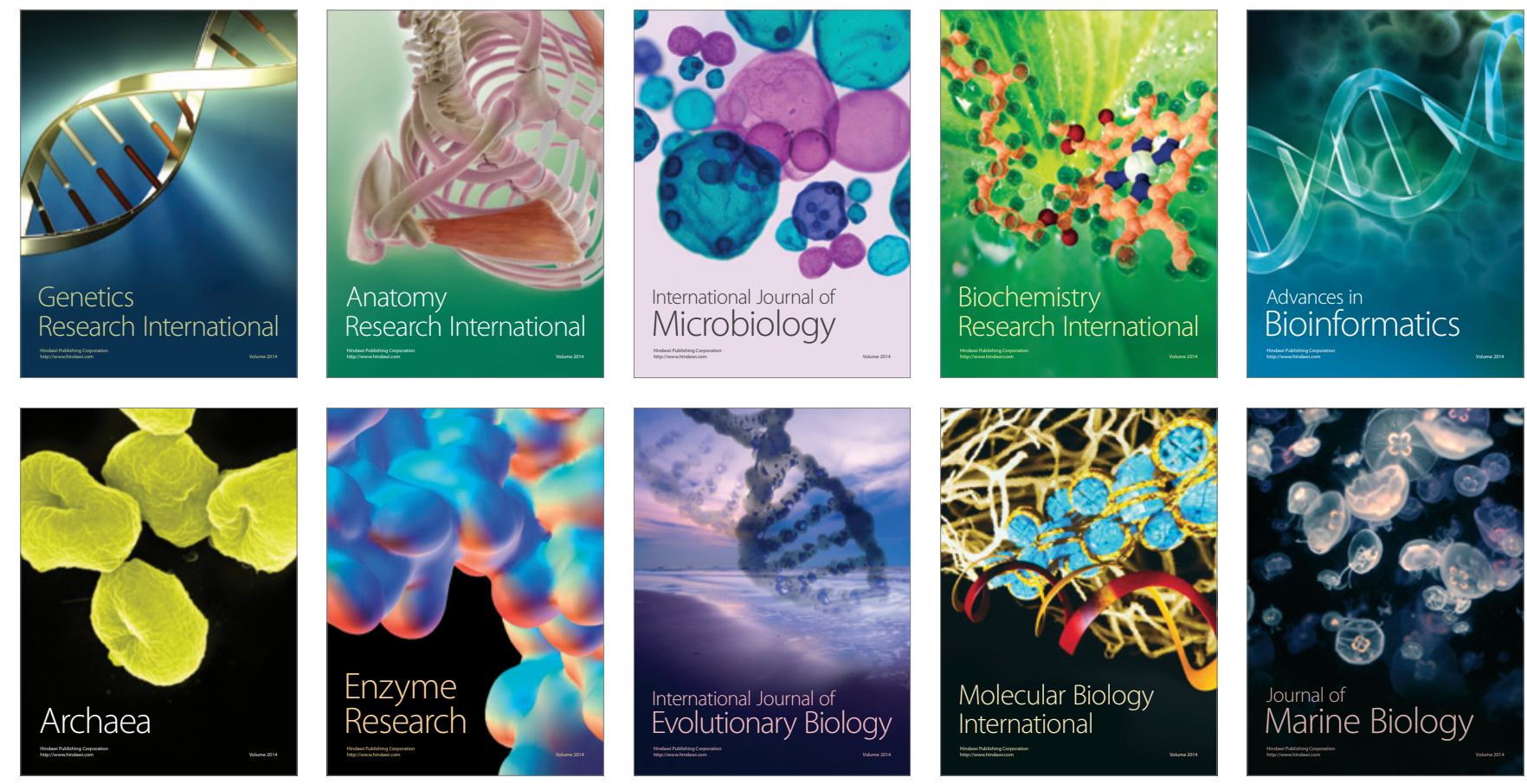